\title{
The influence of stimulus bandwidth on localization of sound in space
}

\author{
ROBERT A. BUTLER and NEIL PLANERT \\ Departments of Surgery [Otolaryngology] and Behavioral Sciences \\ University of Chicago, Chicago, Ilinois 60637
}

\begin{abstract}
The ability of listeners, deprived of prominent interaural time and intensity cues, to locate noise bands differing in width was investigated. To minimize binaural cues, we placed the sound source at various positions in the median sagittal plane. To eliminate binaural cues, we occluded one ear. The stimuli consisted of broadband noise and bands of noise centered at $8.0 \mathrm{kHz}$. The width of the latter ranged from 1.0 to $6.0 \mathrm{kHz}$. The results from seven listeners showed that localization proficiency for sounds in the median sagittal plane decreased with decreases in bandwidth for both binaural and monaural listening conditions. This function was less orderly for monaural localization of horizontally positioned sounds. Another consequence of a reduction in bandwidth was an increasing tendency of listeners to select certain loudspeakers over others as the source of the sound. A previous finding showing that localization of sound in the median sagittal plane is more accurate when listening binaurally rather than monaurally was confirmed.
\end{abstract}

Localization of sound in space is achieved with the aid of interaural time and intensity differences. But they are not essential for reasonably accurate performances on the task. Monaural localization has been demonstrated repeatedly in the horizontal plane (HP) as well as in the median sagittal plane (MSP). One question that frequently arises is what are the cues which govern these monaural localization judgments? For the HP localization task, head movements. which lead to a maximum stimulation of the functioning ear, thereby indicating the segment of the arc from which the sound originates, certainly contribute toward accurate performances. Listeners must merely discriminate between successive changes in loudness. A somewhat less obvious cue for monaural localization is the spectrum of the sound. The experimental data necessary for an adequate description of the role of spectral cues in monaural localization have yet to be collected, but two stimulus conditions prerequisite for accurate monaural performances can be stated, at least, tentatively: (1) the spectrum must be complex, and (2) it must contain the higher audio frequencies. Sinusoids and low-pass noise cannot be located without the benefit of prominent binaural time and intensity cues. Data on localization of sinusoids can be found in the papers of Angell and Fite (1901). Butler (1971). Pratt (1930), Roffler and Butler (1968a), and Trimble (1934). Data on low-pass noise localization are in the papers of Butler (1969). Gardner and Gardner (1973), and Rotfler and Butler (1968b).

The pinnae modify the sound spectrum differentially, depending on the angular relation

This research was supported in part by NINDS Grants NS-11154 and NS-03358 of the USPHS. between the sound source and the interaural axis. SPL measurements for the higher frequencies (ca $>5.0 \mathrm{kHz})$ are most affected by, changes in the position of the sound source. (See Shaw, 1975, for the most recent statement on these physical measurements.) Since, as was mentioned earlier, the stimulus must be complex before it can be localized monaurally, it seems reasonable to assume that the CNS may be comparing the relative amount of acoustic energy among the relevant constituent frequencies as the sound is moved about. One would anticipate, therefore, that accuracy in localization would improve as the complexity of the sound-bandwidth-was increased. This expectation led to the experiments reported below. With the center frequency kept constant, bandwidth was varied over a wide range and observers were requested to locate these stimuli when the binaural cues were either absent or minimal.

\section{METHOD}

The ability of listeners to locate sound was tested in a sound-treated room in which the walls and ceiling were covered with acoustically absorbent material and the floor was carpeted. The sounds were generated by 4-in.-diam KLH loudspeakers. Each loudspeaker was housed in a wooden cabinet, $6^{1 / 4} \times 6^{1 / 4} \times 4$ in., and could be identified by a number ( 1 through 5 ). The task of the listeners was to call out the number of the loudspeaker from which the sound appeared to originate. They were asked not to move their heads. and to assist them in this regard. a headrest was mounted on the back of the listener's chair.

Both broadband and narrowband noise served as stimuli. The narrowband noise was centered arithmetically around $8.0 \mathrm{kHz}$ and was characterized by a sharp $\mathrm{dB} /$ octave drop on either side of its 3 -dB down point. This was achieved by means of a balanced modulator circuit which multiplied an $8.0-\mathrm{kHz}$ sinusoid by a low-pass noise. A Hewlett-Packard audio oscillator (Model 901A) generated the sinusoid. Low-pass noise was produced by connecting 
Table 1

An Outline of the Experimental Conditions Used in This Study

\begin{tabular}{|c|c|c|c|c|}
\hline Part & Plane & $\begin{array}{c}\text { oudspeak } \\
\text { Separa- } \\
\text { tion } \\
\text { (deg) }\end{array}$ & $\begin{array}{l}\text { Listening } \\
\text { Condition }\end{array}$ & Bandwidth (kHz) \\
\hline 1 & MSP & 15 & Binaural & $\begin{array}{r}\mathrm{BB} ; 6.0 ; 5.0 ; 4.0 ; 3.5 ; 3.0 \\
2.5 ; 2.0 ; 1.5 ; 1.0\end{array}$ \\
\hline 2 & MSP & 15 & Monaural & $\mathrm{BB} ; 6.0 ; 5.0 ; 4.0 ; 3.0 ; 2.0$ \\
\hline 3 & HP & 15 & Monaural & $\mathrm{BB} ; 6.0 ; 5.0 ; 4.0 ; 3.0 ; 2.0$ \\
\hline 4 & MSP & 7.5 & Binaural & $\mathrm{BB} ; 6.0 ; 5.0 ; 4.0 ; 3.0 ; 2.0$ \\
\hline
\end{tabular}

Note $-B B=$ broadband

the output of a Grason-Stadler noise generator (Model 901A) to two cascaded SKL filters. The width of the noise band was dependent on the cut-off frequency of the low-pass noise. Specifically, it was twice that frequency. A somewhat more detailed description of the stimulus generated in this manner was published recently by Ruggero (1973), who provided us with the circuitry.

Table 1 describes the plan of the experiment. In Part 1, subjects were asked to locate sounds emanating from the MSP when listening binaurally. The loudspeakers were separated by $15 \mathrm{deg}$, center-to-center, with the lower loudspeaker being - $30 \mathrm{deg}$ and the upper $+30 \mathrm{deg}$ re aural axis, respectively. The loudspeaker arrangement remained the same for Part 2. Subjects, however. listened only with their right ears. Monaural (right ear) listening conditions also applied to Part 3, although here the loudspeakers were arranged in the HP. Again, they were separated by $15 \mathrm{deg}$; the arc extended from 0 to $60 \mathrm{deg}$ azimuth. To establish the monaural conditions for Parts 2 and 3, a Mine Safety Appliance (MSA) ear insert was placed in the subject's left ear canal, then a MSA earmuff was placed over the same ear and fixed by means of an elastic band strapped around the head. Care was taken to prevent the band from touching the pinna of the unoccluded ear since pinna distortion could have further impaired localization performance. To check on the integrity of the left-ear occlusion, we requested the listener, after each block of 25 trials, to place his or her index finger firmly against the ear canal entrance of the right, i.e., unoccluded, ear. Then we presented that stimulus currently under investigation and asked whether it was heard. In nearly every instance it was not. When the integrity of the occlusion was in question, data for the preceding 25-trial block were discarded, the ear insert and muff were refitted, and the block was repeated. The loudspeakers were again positioned in the MSP for Part 4 of the study. This time, however, they were separated from one another by only $7.5 \mathrm{deg}$ and the array extended from -15 to +15 deg re aural axis. Subjects listened binaurally.

Dependence on the MSA muff and ear insert for establishing a condition in which subjects were locating monaurally required that we use a stimulus intensity which (1) would be below the threshold of hearing for the ear being blocked and, at the same time, (2) would be clearly audible. With this in mind, we chose to present the broadband noise at $30 \mathrm{~dB}$ SL re binaural listening and the various narrowband noises at a loudness level approximately equal to that of the broadband noise. The attenuation settings for the different narrowband noises were derived from repeated equal-loudness judgments carried out prior to the experiment by the two experimenters. The same attenuation settings used for monaural conditions were used also for binaural conditions. Stimulus rise-fall time was $10 \mathrm{msec}$; stimulus duration was 500 msee. Stimuli were presented once a second until the listener made a location judgment.

A test session consisted of 100 trials, 50 for one bandwidth and 50 for another. The bandwidths listed in Table 1 were presented a total of 100 trials. Each loudspeaker generated the various stimuli an equal number of times. The order in which the different loudspeakers were activated as well as the order of bandwidth presentation were haphazard.
The proficiency of individual performances was evaluated by means of chi-square tests. The data on location judgments for each subject on each bandwidth were transformed into error scores. An error score of " 0 " indicated that the loudspeaker which generated the stimulus was identitied correctly. An error score of " 1 ," " 2 ," or " 3 ," etc, signified that the listener's location judgment was, respectively, once, twice, or thrice, etc., removed from the correct loudspeaker. When treating the data in this manner, there are two ways in which a nonchance distribution of error scores can take place. One results from accurate localization which would reduce or eliminate the total error score, depend ing, of course, on the degree of accuracy. The other results from a judgmental bias. A bias toward selecting, as the source of the sound, loudspeakers at or adjacent to the middle of the array would lead to a decrease, and a bias toward either extreme of the array would lead to an increase in the total error score re that arising from chance distribution. With this in mind, a second chi-square test was run to check on the presence of biases. Since, for each bandwidth, all loudspeakers served as the sound source an equal number of times, viz, 20, the test determined whether the distribution of loudspeaker selection differed significantly from a rectangular distribution. Confidence levels were set at $p<.01$.

Listeners participated for pay. Admittance as a paid observer was contingent on (1) hearing acuity with in $15 \mathrm{~dB}$ of audiometric zero (ISO; see Davis \& Kranz, 1964) for the frequencies 0.25 through $8.0 \mathrm{kHz}$, and (2) passing a screening test for proficiency in locating sound in space. This test consisted of 50 trials on which broadband noise was to be located in the MSP. Success was defined as having a total error score less than the median total error score calculated for a group of 50 normal hearing listeners who were tested under comparable conditions (Butler, 1970). Seven listeners were selected. Prior to formal testing, they were given an additional 50 trials, under conditions of binaural listening, on each of the bandwidths listed in Part 1. This procedure served to reduce the influence of practice on the results of the main study. Another listener (L.B.) was given an abbreviated version of Parts 2 and 3-50 trials on each bandwidth. He had had a unilateral hearing loss for over 20 years and had participated in previous localization studies. His data were compared with those of the group.

\section{RESULTS}

Figure 1a summarizes the listeners' performances with respect to total error scores on Parts 1, 2, and 3. Theoretically, a total error score of 160 would result from a random selection of loudspeakers. Two points warrant mention: (1) The total error scores decreased with increases in stimulus bandwidth, although this function was less orderly for the HP localization task. (2) When locating sounds in the MSP, total error score under binaural listening conditions was consistently less than that recorded under monaural conditions. The error score data for Part 4 are plotted separately (see Figure 1b). The loudspeakers, in this situation, were closer together; hence, there is no satisfactory way to compare directly the data from Part 4 with those of the other parts. The only point to be made is that performance improved at the wider bandwidths. The Part 4 data are more useful in another connection-judgmental biases-which will be treated later.

As mentioned in the Methods section, error score data cannot be interpreted unequivocally as an index of proficient localization, since a bias in loudspeaker 


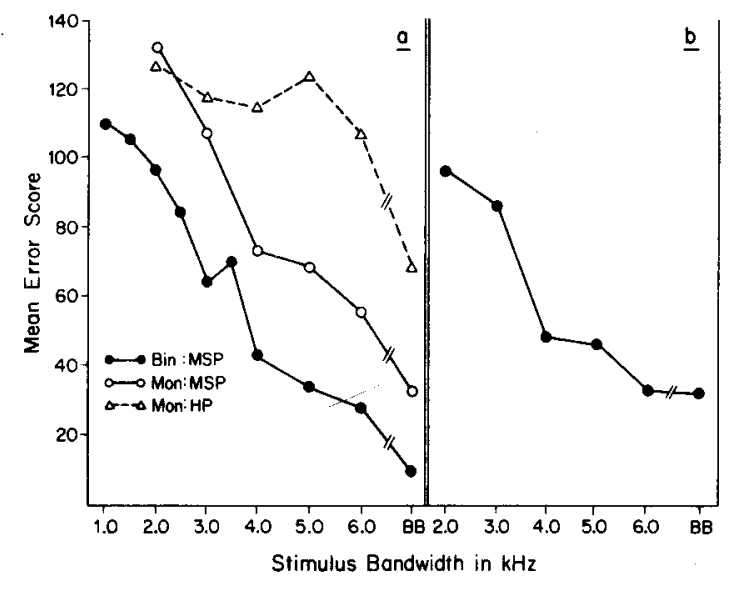

Figure 1. (a) Mean error score as a function of stimulus bandwidth for those experimental conditions in which the loudspeakers were 15 deg apart (Parts 1, 2, and 3). Bin and Mon refer to binaural and monaural listening, respectively. (b) A plot of Part 4 data in which 7.5 deg separated adjacent loudspeakers.

selection can influence the distribution in a statistically significant manner. Hence, an analysis of individual performances was carried out from which we arrived at two levels of localization proficiency and one level which clearly indicated a failure to locate. The highest level, Level 1 , can be characterized by an error score distribution which differed significantly from chance expectation. but a distribution of loudspeaker selection which did not differ signiticantly from a rectangular distribution. In other words, the listener was, indeed, locating the sound source for that particular bandwidth. Level 2 is that in which both error score distribution and the distribution of loudspeaker selection differed significantly from the theoretical distribution. The data in this category needed further analysis before one could account adequately for the localization performances because a judgmental bias was being expressed. At Level 3, the error score distribution did not differ from a distribution based on chance, and, although the distribution of loudspeaker selection frequently deviated from that expected from a random choice, it mattered little. The listener simply was not locating the bandwidth in question.

The number of listeners who performed at Levels 1 and 2 is listed in Table 2 . These results of individual chi-square tests are consonant with the performance trends based on total error scores shown in Figure 1. Individual performances improved with wider bandwidths. What arises from this test-by-test analysis otherwise concealed by pooling the data is that a bias in the selection of loudspeakers becomes more and more prevalent as the localization task becomes increasingly difficult. But first let us consider only Level 1 performances. With the exception of the monaural HP localization task (Part 3), most listeners performed well on bandwidths of $4.0 \mathrm{kHz}$ and above. Upon closer examination, however, one is struck by the large individual differences with respect to the narrowest bandwidth that could still be located proficiently. In Part 1, for example, one listener located accurately all bandwidths $1.0 \mathrm{kHz}$ and above; another performed comparably on bandwidths of $2.0 \mathrm{kHz}$ and above. At the other extreme, one listener performed proficiently only on bandwidths of $5.0 \mathrm{kHz}$ and above. Those two listeners, incidentally, who excelled on Part 1 also performed well on Part 4 (another binaural condition), but they did not distinguish themselves from the group on Parts 2 or 3 (monaural conditions). For normal hearing listeners, locating sounds monaurally is, at the very least, a sudden departure from the everyday listening experience. It is in this connection that the data of L.B. are informative. Admittedly, comparing his data with the group mean is a gross procedure, but it is of interest to note that L.B.'s performance excelled that of the group only when the stimuli were positioned along the horizontal plane. In somewhat more detail, L.B. performed at Level 1 on broadband noise and noise bands 3.0 and $4.0 \mathrm{kHz}$ in width (Part 3), but, curiously, he failed to demonstrate a comparable level of proficiency for the $5.0-$ and $6.0 \cdot \mathrm{kHz}$ bandwidths. $\mathrm{He}$ attained Level 1 proficiency during Part 2 only when the bandwidth was $6.0 \mathrm{kHz}$. Figures $2 \mathrm{a}$ and $2 \mathrm{~b}$ illustrate L.B.'s performance compared with the group performance on Parts 2 and 3 , respectively. Since L.B. was given only 50 trials on each bandwidth. the right-hand ordinate is scaled accordingly.

We turn now to Level 2 performances. What appeared to be proficient localization, as defined by the distribution or error scores, was confounded by judgmental biases. The bias expressed during the

Table 2

Instances of Level 1 and Level 2 Performances on the Various Bandwidths for Each Part of the Experiment

\begin{tabular}{|c|c|c|c|c|c|c|c|c|c|c|c|c|c|c|c|c|c|c|c|c|}
\hline & \multicolumn{2}{|c|}{ BB } & \multicolumn{2}{|c|}{6.0} & \multicolumn{2}{|c|}{5.0} & \multicolumn{2}{|c|}{4.0} & \multicolumn{2}{|c|}{3.5} & \multicolumn{2}{|c|}{3.0} & \multicolumn{2}{|c|}{2.5} & \multicolumn{2}{|c|}{2.0} & \multicolumn{2}{|c|}{1.5} & \multicolumn{2}{|c|}{1.0} \\
\hline & 1 & 2 & 1 & 2 & 1 & 2 & 1 & 2 & 1 & 2 & 1 & 2 & 1 & 2 & 1 & 2 & 1 & 2 & 1 & 2 \\
\hline $\begin{array}{l}\text { Part } 1 \\
\text { Part } 2 \\
\text { Part } 3 \\
\text { Part } 4\end{array}$ & $\begin{array}{l}6 \\
5 \\
2 \\
6\end{array}$ & $\begin{array}{l}1 \\
2 \\
5 \\
1\end{array}$ & $\begin{array}{l}7 \\
5 \\
1 \\
7\end{array}$ & $\begin{array}{l}0 \\
1 \\
4 \\
0\end{array}$ & $\begin{array}{l}6 \\
3 \\
0 \\
6\end{array}$ & $\begin{array}{l}1 \\
3 \\
3 \\
1\end{array}$ & $\begin{array}{l}5 \\
4 \\
0 \\
6\end{array}$ & $\begin{array}{l}2 \\
2 \\
4 \\
1\end{array}$ & 3 & 4 & $\begin{array}{l}4 \\
1 \\
1 \\
3\end{array}$ & $\begin{array}{l}2 \\
4 \\
3 \\
3\end{array}$ & 2 & 4 & $\begin{array}{l}3 \\
0 \\
0 \\
1\end{array}$ & $\begin{array}{l}3 \\
3 \\
3 \\
4\end{array}$ & 1 & 3 & 1 & 3 \\
\hline
\end{tabular}

Note-Levels are indicated immediately below each bandwidth. BB refers to broadband noise. All other bandwidths are in kilohertz. 


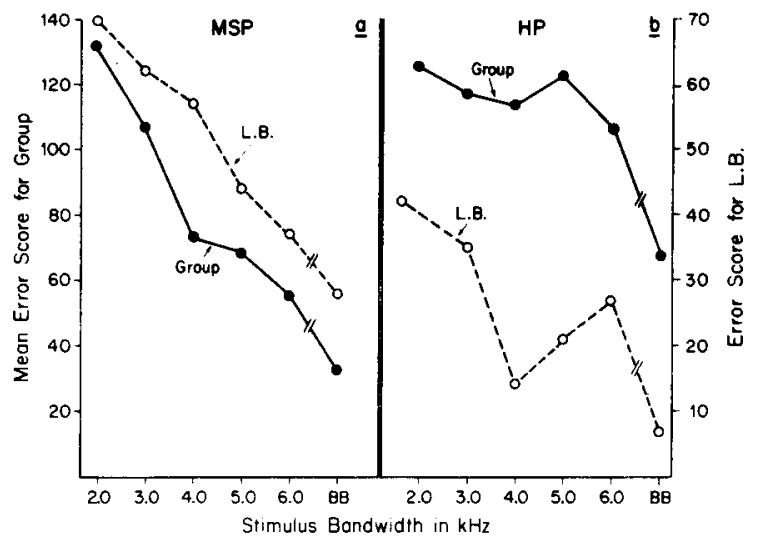

Figure 2. (a) Comparison of L.B.'s error score with group mean for Part 2-monaural localization in MSP. (b) Comparison of L.B.'s error score with group mean for Part 3-monaural localization in HP.

monaural HP localization task (Part 3) was a displacement of the sound toward the unoccluded ear; hence, rarely was Loudspeaker No. 1 (0-deg azimuth) chosen. And at narrower bandwidths Loudspeaker No. 2 (15-deg azimuth) was seldom selected. When locating sounds in the MSP, the bias expressed in Part 1 was that of choosing Loudspeakers 2, 3, and 4 at the expense of Loudspeakers 1 and 5. Part 4 of the experiment was designed to increase the difficulty of the task, but the data obtained by placing the loudspeakers closer together provided an answer to an interesting question concerning judgmental biases. To wit. under conditions of restricted bandwidths, did listeners select a loudspeaker as the origin of the sound because of its relative position within the array of loudspeakers, or because of its elevation re the aural axis? Is an $8.0-\mathrm{kHz}$-centered narrowband noise, in other words, perceived at a specific position in space which is not influenced by the response options available to the listener? Based on localization responses to the narrowest bandwidth common to both parts 1 and $4-2.0 \mathrm{kHz}$-it appears that it was the relative position of the loudspeaker which influenced location judgments, not its elevation. Figure 3 shows, for Level 2 performances, the distribution of loudspeaker selection when locating the $2.0-\mathrm{kHz}$ bandwidth with the loudspeakers being either 15 or $7.5 \mathrm{deg}$ apart. Note that the distribution of loudspeaker selection is similar when frequency of loudspeaker choice is plotted against the ordinal position of the loudspeaker. This finding is incompatible with the notion that, when the bandwidth is appreciably confined, the stimulus appears to originate from a specitic elevation.

There remains still another question concerning judgmental biases; vi\% were the biases primarily responsible for those error score distributions which differed from that attributable to chance? Since the tendency was to cluster the loudspeaker choices toward the center of the array, thereby reducing the likelihood of committing large errors, the question warrants consideration. Perhaps listeners when exhibiting biases were indeed not localizing. Inspection of the MSP data suggested, however, that nearly all listeners could still detect a change in the up-down location of a sound source from one trial to the next. provided it involved nonadjacent loudspeakers. The listener may not have selected the "correct" loudspeaker, but he or she usually selected one which was in the appropriate vertical direction re the loudspeaker activated on the previous trial. This level of localization performance obviously serves to reduce the size of the error score, and one can infer, with reasonable assurance. that those listeners whose error score distribution differed from a chance distribution were. in the majority of instances. discriminating between the relative location of sound source. The same generalization can be made for the HP localization performances which, as shown in Table 2. were pervaded by biases.

There were 37 instances of Level 3 performance, 26 of which showed a strong judgmental bias.

\section{DISCUSSION}

This experiment sought to answer the question of how complex, as defined in terms of bandwidth, a sound must be in order to be located proficiently without the benefit of binaural cues. Upon analysis of the data. it became apparent that there were two levels of proficiency. At one level, the error scores were small because listeners were doing well at locating sound sources; at the other. localization data

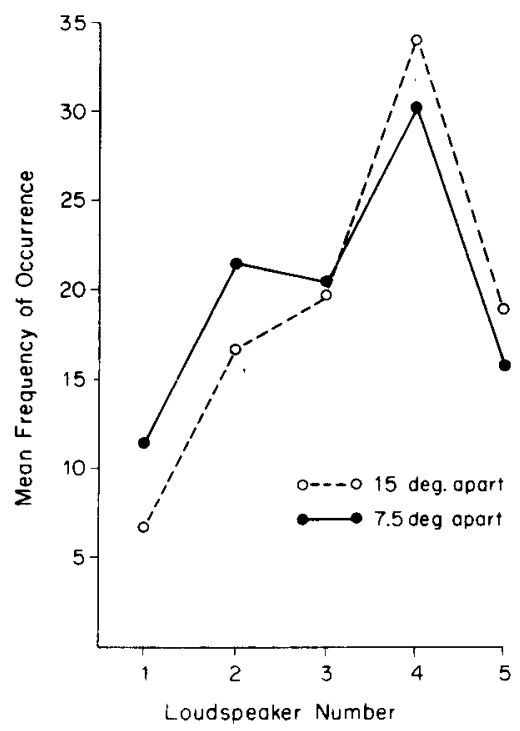

Figure 3. Frequency with which various loudspeakers were selected as the source of a $2.0-\mathrm{kHz}$ bandwidth. Loudspeaker No. 1 was at the top of the array; No. 5 was at the bottom. 
were confounded by judgmental biases. The question posed at the beginnir: of this paragraph should be answered in terms of the more easily interpretable data categorized under Level 1 . All in all, instances of Level 1 performances under monaural listening conditions were few. When they did occur, there were usually associated with the wider bandwidths a finding in accord with the supposition that the CNS compares the relative distribution of acoustic energy among the various frequencies as a cue for a sound's position in space. Bandwidths which encompass more of the higher audio frequencies simply provide more complete spectral cues.

The data for Parts 1 and 4 (MSP localization tasks) more clearly attest to the importance of bandwidth in attaining Level 1 proficiency. But the situation here is somewhat different; subjects were listening binaurally. Why should that matter if each ear is receiving the same acoustic input from the MSP positioned sounds? Apparently they are not. Searle (1973) reported on the frequency response curves measured at each ear canal in the same subject when the sound originated from various MSP elevations. At the higher audio frequencies $(>8.0 \mathrm{kHz})$, the frequency response curves differed markedly from one another. Clearly, this event provides an interaural difference cue with respect to the spectrum of the stimulus, which may help explain why binaural performance is superior to monaural performance on MSP localization tasks-a tinding reported by Butler (1969), corroborated by Gardner (1973), and again by the results of Part 1 when compared with Part 2 in the present study. Perhaps those two listeners who excelled when locating the narrower bandwidths binaurally but who failed to distinguish themselves when locating monaurally may, by virtue of asymmetries between pinnae, have been either (1) receiving a more pronounced binaural spectral difference cue. or (2) utilizing this possible binaural cue more effectively.

Considerable space was devoted to judgmental biases in the Results section. Because there were nearly as many instances of biases as there were instances free from biases. a few comments seem to be in order. In the face of inadequate or even misleading auditory cues. localization judgments are influenced by the total testing situation. A judgment is expected of the listener, and he or she makes this judgment not only on the present acoustic evidence, but on the entire history of perceiving sound in space. To paraphrase Gregory (1966), perception is an active process filling the gaps in sensory information so as to make the most sense of what is almost always an ambiguous sensory input. The perceptual response, in briet. is to make the event more probable. In tracing the course of the judgmental biases as the bandwidth became progressively restricted, the tendency was to choose a single loudspeaker or two adjacent loudspeakers more and more often as the source of the sounds. These choices happened to be clustered about the center of the loudspeaker array. It is suggested that this pattern of choices did not reflect a deliberate strategy adopted to minimize the size of the localization error. Rather, it is as if a sound of restricted bandwidth which was perceived as coming from, say. Loudspeaker No. 4 in the binaural MSP localization task, could not be expected, on a subsequent trial, to originate from a loudspeaker as far removed as No. 1. The spectral cues furnished by a bandwidth of only 2.0 or $3.0 \mathrm{kHZ}$ are impoverished, and the spectrum is not sufficiently modified when the sound source originates from widely different positions. In the monaural localization, the auditory information provided by the narrow bandwidths was not only inadequate for proficient localization, it was misleading. But our interpretation in terms of making probable an improbable event still applies. A sound originating from a loudspeaker positioned straight ahead would not be expected to stimulate just one ear. Such a difference in binaural loudness cannot arise even when the sound is opposite the open ear, but it is more likely to do so. Hence, in the monaural task, the sound was displaced toward the unoccluded ear. In the monaural MSP localization task, some listeners reported a displacement toward the open ear, but since there was no visible loudspeaker in that region, these listeners had to "transfer" the sound source to the vertical array of loudspeakers in order to call out the number of the loudspeaker at the corresponding elevation. This confusion probably contributed toward the impaired performances. What about those few cases where neither error score distribution nor distribution of loudspeaker selection differed from that expected by chance? These, too, involved the more restricted bandwidths. We can only speculate that the listeners, having experienced on tests employing the broader bandwidths that the sound could come from any loudspeaker and assuming that this rule still held, simply discounted the acoustic input and guessed.

\section{REFERENCES}

Angell. J. R.. \& Fite. W. The monaural localization of sound. Psychological Review, 1901. 8. 225-246.

Butler. R. A. Monaural and binaural localization of noise bursts vertically in the median sagittal plane. Journal of Auditory Research, 1969, 3. 230-235.

BUTLER. R. A. The effect of hearing impairment on locating sound in the vertical plane. International Audiology. 1970 , 9. 117-126.

Butler, R. A. The monaural localization of tonal stimuli. Perception \& Psychophysics. 1971. 9. 99-101.

Davis. H.. \& Kranz, F. W. The international standard reference zero for pure-tone audiometers and its relation to the evaluation of impairment of hearing. Journal of Speech and Hearing Research, 1904, 7, 7-16. 
GaroNer. M. B. Some monaural and binaural facets of median plane localization. Journal of the Acoustical Sociern of America, 19-3, 54. 1489-1495.

Gardner. M. B.. \& Gardner, R. S. Problem of localization in the median plane: Effect of pinnae carity occlusion. Journal of the Acoustical Societr of America. 19-3. 53. $400-408$.

Gregory. R. A. Elye und brain. New York: McGraw-Hill. $1 \%$ to.

Pratt. C. C. The spatial character of high and low tones. Journal of Experimental Psichology. 1930. 13. 2-8-285.

Roffler. S. K.. \& Bctier. R. A. Factors that influence the localization of sound in vertical plane. Joumal of Acoustical Socit't of America. 1968. 43. 1255-1259. (a)

Roffler. S. K.. \& Bltler. R. A. Localization of tonal stimuli in the vertical plane. Joumal of the Acoustical Socitry of America. 1968. 43. 1200-1206. (b)
RLGgero. M. A. Response to noise of auditory nerve fibers in the squirrel monkey. Journal of Neurophysiology. 1973. 36. $504.58^{-}$

SeARLe. C. L. Cues required for externalization and vertical localization. Jourmal of the Acoustical Society of America. 19-3. 54. 308(A).

Shak. E. A. G. The external ear. In W. D. Keidel and W. D. Neft (Eds.). Hundbook of sensong physiology (Vol. 5. No. 2). Berlin: Springer-Verlag. 1975.

Trimble. $O$. S. Loxilization of sound in the anterior posterior and vertical Jimensions of auditory space. Bribth Journal of Psichologi. 1434, 24, 320-334.

(Received for publication July 1. 1974: revision accepted November 25,1974 ,) 